\title{
Some remarks about the existence of coupled $g$-coincidence points
}

\author{
Inci M Erhan ${ }^{1}$, Antonio-Francisco Roldán-López-de-Hierro² and Naseer Shahzad ${ }^{3 *}$
}

\section{"Correspondence:}

nshahzad@kau.edu.sa

${ }^{3}$ Department of Mathematics, King

Abdulaziz University, P.O. Box 80203

Jeddah, 21589, Saudi Arabia

Full list of author information is

available at the end of the article

\begin{abstract}
Very recently, in a series of subsequent papers, Nan and Charoensawan introduced the notion of $g$-coincidence point of two mappings in different settings (metric spaces and G-metric spaces) and proved some theorems in order to guarantee the existence and uniqueness of such kind of points. Although their notion seems to be attractive, in this paper, we show how this concept can be reduced to the unidimensional notion of coincidence point, and how their main theorems can be seen as particular cases of existing results. Moreover, we prove that the proofs of their main statements have some gaps.
\end{abstract}

\section{Introduction}

After the appearance of the works by Turinici [1] and, subsequently, by Ran and Reurings [2] and Nieto and Rodríguez-López [3] in partially ordered metric spaces, the branch of fixed point theory devoted to the study of existence and uniqueness of coupled, tripled, quadrupled, and, in general, multidimensional fixed points has attracted much attention. Unfortunately, many of the presented high-dimensional results become simple consequences of their corresponding unidimensional versions (see [4-12] and references therein).

Very recently, Nan and Charoensawan [13] introduced the notion of $g$-coincidence point of two mappings $F, H: X \times X \rightarrow X$, and proved existence and uniqueness theorems of such kind of points. In this paper, we show that their results can be seen as simple consequences of existing unidimensional results. The same commentaries about their results can also be done for the statements introduced by the same authors in [14].

In order not to enlarge this short-note unnecessarily, we only include some basic preliminaries. The rest of definitions and basic facts can be found in the mentioned papers.

\section{Preliminaries}

In the sequel, we denote by $\mathbb{N}=\{0,1,2, \ldots\}$ the family of all nonnegative integers. Let $X$ denote a nonempty set and, given $n \in \mathbb{N}, n \geq 2$, let $X^{n}$ be the product space $X \times X \times \stackrel{(n)}{\cdots} \times X$. Henceforth, $T, g, F$, and $H$ stand for mappings as follows: $T, g: X \rightarrow X$ and $F, H: X^{2} \rightarrow X$. Consider the following kind of control functions.

Definition 2.1 Let $\Phi$ be the family of all functions $\varphi:[0, \infty) \rightarrow[0, \infty)$ satisfying

$\left(\mathcal{P}_{1}\right) \varphi(t)<t$ for all $t>0$;

$\left(\mathcal{P}_{2}\right) \lim _{s \rightarrow t^{+}} \varphi(s)<t$ for all $t>0$.

(c) 2015 Erhan et al.; licensee Springer. This is an Open Access article distributed under the terms of the Creative Commons Attribution License (http://creativecommons.org/licenses/by/4.0), which permits unrestricted use, distribution, and reproduction in any medium, provided the original work is properly credited. 
Remark 2.1 (1) Notice that the previous properties do not determine the value $\varphi(0)$, which can be arbitrary. For instance, given $a>0$, the function $\varphi_{a}:[0, \infty) \rightarrow[0, \infty)$, defined, for all $t \in[0, \infty)$, by

$$
\varphi_{a}(t)= \begin{cases}a, & \text { if } t=0, \\ t / 2, & \text { if } t>0,\end{cases}
$$

belongs to $\Phi$. However, $\varphi_{a}(0)=a>0$.

(2) By $\left(\mathcal{P}_{1}\right)$, it is clear that $\lim _{t \rightarrow 0^{+}} \varphi(t)=0$ for all $\varphi \in \Phi$. However, this property does not imply the following one: if $\left\{a_{n}\right\},\left\{b_{n}\right\} \subset[0, \infty)$ verify that $a_{n} \leq \varphi\left(b_{n}\right)$ for all $n \in \mathbb{N}$ and $\left\{b_{n}\right\} \rightarrow 0$, then $\left\{a_{n}\right\} \rightarrow 0$. For instance, given $a>0$, if we define $a_{n}=a$ and $b_{n}=0$ for all $n \in \mathbb{N}$, then $a_{n}=a=\varphi_{a}(0)=\varphi_{a}\left(b_{n}\right)$ for all $n \in \mathbb{N}$ and $\left\{b_{n}\right\} \rightarrow 0$, but $\left\{a_{n}\right\} \rightarrow a \neq 0$.

The following definitions and theorem can be found in [15].

Definition 2.2 Let $T, g: X \rightarrow X$ be two mappings and let $\mathcal{M} \subseteq X^{2}$ be a subset. We will say that $M$ is:

- $(T, g)$-closed if $(T x, T y) \in \mathcal{M}$ for all $x, y \in X$ such that $(g x, g y) \in \mathcal{M}$;

- $(T, g)$-compatible if $T x=T y$ for all $x, y \in X$ such that $g x=g y$.

Definition 2.3 We will say that a subset $\mathcal{M} \subseteq X^{2}$ is transitive if $(x, y),(y, z) \in \mathcal{M}$ implies that $(x, z) \in \mathcal{M}$.

Definition 2.4 Let $(X, d)$ be a metric space and let $\mathcal{M} \subseteq X^{2}$ be a subset. We will say that $(X, d, \mathcal{M})$ is regular if for all sequence $\left\{x_{n}\right\} \subseteq X$ such that $\left\{x_{n}\right\} \rightarrow x$ and $\left(x_{n}, x_{n+1}\right) \in \mathcal{M}$ for all $n \in \mathbb{N}$, we have $\left(x_{n}, x\right) \in \mathcal{M}$ for all $n \in \mathbb{N}$.

Definition 2.5 Let $(X, d)$ be a metric space, let $\mathcal{M} \subseteq X^{2}$ be a subset and let $x \in X$. A mapping $T: X \rightarrow X$ is said to be $\mathcal{M}$-continuous at $x$ if for all sequence $\left\{x_{n}\right\} \subseteq X$ such that $\left\{x_{n}\right\} \rightarrow x$ and $\left(x_{n}, x_{n+1}\right) \in \mathcal{M}$ for all $n \in \mathbb{N}$, we have $\left\{T x_{n}\right\} \rightarrow T x$. $T$ is $\mathcal{M}$-continuous if it is $\mathcal{M}$-continuous at each $x \in X$.

The reader can compare the previous notion with the concept of 'nondecreasingcontinuity' introduced in [16].

Remark 2.2 Every continuous mapping is also $\mathcal{M}$-continuous, whatever $\mathcal{M} \subseteq X^{2}$.

Definition 2.6 Let $(X, d)$ be a metric space and let $\mathcal{M} \subseteq X^{2}$ be a subset. Two mappings $T, g: X \rightarrow X$ are said to be $(O, \mathcal{M})$-compatible if

$$
\lim _{n \rightarrow \infty} d\left(g \operatorname{Tx}, \operatorname{Tg} x_{n}\right)=0
$$

provided that $\left\{x_{n}\right\}$ is a sequence in $X$ such that $\left(g x_{n}, g x_{n+1}\right) \in \mathcal{M}$ for all $n \geq 0$ and

$$
\lim _{n \rightarrow \infty} T x_{n}=\lim _{n \rightarrow \infty} g x_{n} \in X
$$

Remark 2.3 If $T$ and $g$ are commuting (that is, $\operatorname{Tg} x=g T x$ for all $x \in X$ ), then they are also $(O, \mathcal{M})$-compatible, whatever $\mathcal{M}$.

The main result in [15] was the following one. 
Theorem 2.1 (Karapınar et al. [15, Theorem 33]) Let $(X, d)$ be a complete metric space, let $T, g: X \rightarrow X$ be two mappings such that $T X \subseteq g X$ and let $\mathcal{M} \subseteq X^{2}$ be a $(T, g)$-compatible, $(T, g)$-closed, transitive subset. Assume that there exists $\varphi \in \Phi$ such that

$$
d(T x, T y) \leq \varphi(d(g x, g y)) \quad \text { for all } x, y \in X \text { such that }(g x, g y) \in \mathcal{M} \text {. }
$$

Also assume that, at least, one of the following conditions holds:

(a) $T$ and $g$ are $\mathcal{M}$-continuous and $(O, \mathcal{M})$-compatible;

(b) $T$ and $g$ are continuous and commuting;

(c) $(X, d, \mathcal{M})$ is regular and $g(X)$ is closed.

If there exists a point $x_{0} \in X$ such that $\left(g x_{0}, T x_{0}\right) \in \mathcal{M}$, then $T$ and $g$ have, at least, a coincidence point.

Next, we show that the proof of Theorem 2.1 given by the authors in [15] demonstrates, point by point, a slightly stronger result. To do that, we notice that some of the definitions involved in the last theorem were subtly modified in [17] in the following sense.

Definition 2.7 (See Kutbi et al. [17]) Given two mappings $T, g: X \rightarrow X$, we say that a nonempty subset $\mathcal{M}$ of $X^{2}$ is:

- $g$-transitive if $(g x, g z) \in \mathcal{M}$ for all $x, y, z \in X$ such that $(g x, g y),(g y, g z) \in \mathcal{M}$;

- $(T, g)$-compatible if $T x=T y$ for all $x, y \in X$ such that $g x=g y$ and $(g x, g y) \in \mathcal{M}$.

With respect to the previous definitions, we point out the following remarks.

(1) In the proof of Theorem 2.1 in [15], the hypothesis ' $\mathcal{M}$ is $(T, g)$-compatible' was only used in one subcase of case (c) (the subcase in which there exists some $m_{0} \in \mathbb{N}$ such that $\left.d\left(g x_{m_{0}}, x\right)=0\right)$. Then we can remove it from the general hypotheses of the theorem if we add it to assumption (c).

(2) The notions of $(T, g)$-compatibility in Definitions 2.2 and 2.7 are different. In fact, the notion given in Definition 2.7 is weaker than the concept given in Definition 2.2. However, as the reader can easily check, in the proof of Theorem 2.1 given by the authors in [15], they only used $(T, g)$-compatibility in the sense of Definition 2.7 because they assumed that, in the mentioned subcase, we also have $\left(g x_{m_{0}}, g z\right) \in \mathcal{M}$.

(3) Although the authors assumed that $\mathcal{M}$ is transitive in Theorem 2.1, in fact, they only used that $\mathcal{M}$ is $g$-transitive to guarantee that

$$
\left[\left(g x_{n}, g x_{n+1}\right) \in \mathcal{M} \text { for all } n \in \mathbb{N}\right] \quad \Rightarrow \quad\left[\left(g x_{m}, g x_{n}\right) \in \mathcal{M} \text { for all } m, n \in \mathbb{N}, m<n\right] .
$$

General transitivity is not necessary in Theorem 2.1.

(4) By Remark 2.2, we can suppose that $T$ and $g$ are continuous in case (a) because this condition implies that they are also $\mathcal{M}$-continuous.

As a consequence of the previous commentaries, we deduce that the subtle refinement given in Definition 2.7 shows that the proof of Theorem 2.1 given by the authors in [15] demonstrates, point by point, the following stronger result (in which we use $(T, g)$ compatibility in the sense of Definition 2.7).

Theorem 2.2 Let $(X, d)$ be a complete metric space, let $T, g: X \rightarrow X$ be two mappings such that $T X \subseteq g X$ and let $\mathcal{M} \subseteq X^{2}$ be a $(T, g)$-closed, $g$-transitive subset. Assume that 
there exists $\varphi \in \Phi$ such that

$$
d(T x, T y) \leq \varphi(d(g x, g y)) \quad \text { for all } x, y \in X \text { such that }(g x, g y) \in \mathcal{M} .
$$

Also assume that, at least, one of the following conditions holds.

(a) $T$ and $g$ are $\mathcal{M}$-continuous and $(O, \mathcal{M})$-compatible;

$\left(\mathrm{a}^{\prime}\right) T$ and $g$ are continuous and $(O, \mathcal{M})$-compatible;

(b) $T$ and $g$ are continuous and commuting;

(c) $(X, d, \mathcal{M})$ is regular, $g(X)$ is closed and $\mathcal{M}$ is $(T, g)$-compatible.

If there exists a point $x_{0} \in X$ such that $\left(g x_{0}, T x_{0}\right) \in \mathcal{M}$, then $T$ and $g$ have, at least, a coincidence point.

Remark 2.4 The condition ' $g(X)$ is closed' of assumption (c) was only used to guarantee that $(g(X), d)$ is a complete metric space. As a consequence, the same thesis can be deduced replacing, in case (c), that ' $(X, d)$ is complete and $g(X)$ is closed' by the weaker condition ' $g(X)$ is $d$-complete', and the proof is obtained by verbatim. This argument was already employed, for instance, in the proof of Theorem 34 in [11].

\section{About some coupled $g$-coincidence point theorems in metric spaces}

From now on, $g: X \rightarrow X$ and $F, H: X^{2} \rightarrow X$ will denote arbitrary mappings. In [13], the authors introduced the following notions.

Definition 3.1 An element $(x, y) \in X^{2}$ is called a coupled g-coincidence point of the mappings $F$ and $H$ if $F(x, y)=H(g x, g y)$ and $F(y, x)=H(g y, g x)$.

Definition 3.2 Let $M$ be a subset of $X^{4}$. We say that $M$ is an $\left(H_{g}, F\right)$-closed subset of $X^{4}$ if for all $x, y, u, v \in X$,

$$
\begin{gathered}
(H(g x, g y), H(g y, g x), H(g u, g v), H(g v, g u)) \in M \\
\Rightarrow \quad(F(x, y), F(y, x), F(u, v), F(v, u)) \in M .
\end{gathered}
$$

Definition 3.3 Let $M$ be a subset of $X^{4}$. We say that $M$ satisfies the transitive property if, and only if, for all $x, y, u, v, a, b \in X$,

$$
\begin{aligned}
& (H(x, y), H(y, x), H(u, v), H(v, u)) \in M \quad \text { and } \quad(H(u, v), H(v, u), H(a, b), H(b, a)) \in M \\
& \Rightarrow \quad(H(x, y), H(y, x), H(a, b), H(b, a)) \in M .
\end{aligned}
$$

Definition 3.4 We say that the pair $\{F, G\}$ is $g$-generalized compatible if

$$
\left\{\begin{array}{l}
d\left(F\left(H\left(g x_{n}, g y_{n}\right), H\left(g y_{n}, g x_{n}\right)\right), H\left(g F\left(x_{n}, y_{n}\right), g F\left(y_{n}, x_{n}\right)\right)\right) \rightarrow 0 \quad \text { as } n \rightarrow \infty \\
d\left(F\left(H\left(g y_{n}, g x_{n}\right), H\left(g x_{n}, g y_{n}\right)\right), H\left(g F\left(y_{n}, x_{n}\right), g F\left(x_{n}, y_{n}\right)\right)\right) \rightarrow 0 \quad \text { as } n \rightarrow \infty
\end{array}\right.
$$

whenever $\left\{x_{n}\right\},\left\{g x_{n}\right\},\left\{y_{n}\right\}$, and $\left\{g y_{n}\right\}$ are sequences in $X$ such that

$$
\left\{\begin{array}{l}
\lim _{n \rightarrow \infty} F\left(x_{n}, y_{n}\right)=\lim _{n \rightarrow \infty} H\left(g x_{n}, g y_{n}\right)=t_{1}, \\
\lim _{n \rightarrow \infty} F\left(y_{n}, x_{n}\right)=\lim _{n \rightarrow \infty} H\left(g y_{n}, g x_{n}\right)=t_{2} .
\end{array}\right.
$$


The main theorem in [13] was the following one.

Theorem 3.1 (Nan and Charoensawan [13, Theorem 3.1]) Let $(X, d)$ be a complete metric space and $M$ be a nonempty subset of $X^{4}$. Assume that $g: X \rightarrow X$ is continuous and $F, H: X \times X \rightarrow X$ are two generalized compatible mappings such that $H$ is continuous, and for any $x, y \in X$, there exist $u, v \in X$ such that $F(x, y)=H(g u, g v)$ and $F(y, x)=H(g v, g u)$. Suppose that there exists $\varphi \in \Phi$ such that the following holds:

$$
\begin{aligned}
& d(F(x, y), F(u, v))+d(F(y, x), F(v, u)) \\
& \quad \leq \varphi(d(H(g x, g y), H(g u, g v))+d(H(g y, g x), H(g v, g u)))
\end{aligned}
$$

for all $x, y, u, v \in X$ with $(H(g x, g y), H(g y, g x), H(g u, g v), H(g v, g u)) \in M$.

Also suppose that $F$ is continuous. If there exists $\left(x_{0}, y_{0}\right) \in X$ such that

$$
\left(H\left(g x_{0}, g y_{0}\right), H\left(g y_{0}, g x_{0}\right), F\left(x_{0}, y_{0}\right), F\left(y_{0}, x_{0}\right)\right) \in M
$$

and $M$ is $\left(H_{g}, F\right)$-closed, then there exists $(x, y) \in X \times X$ such that $H(g x, g y)=F(x, y)$ and $H(g y, g x)=F(y, x)$, that is, $F$ and $H$ have a coupled $g$-coincidence point.

Notice that the authors did not established in the statement of the previous theorem that $M$ is transitive in the sense of Definition 3.3, but they used it throughout their proof. The authors also forgot to say that the pair $\{F, H\}$ is $g$-generalized compatible (they only mentioned generalized compatible pairs, which correspond to a different notion that they had previously commented in [13]). Next, we show that this is not a new result.

Theorem 3.2 Theorem 3.1 (including the facts that ' $M$ is transitive' and replacing the hypothesis ' $F, H: X \times X \rightarrow X$ are two generalized compatible mappings' by ' $F, H: X \times X \rightarrow X$ are two g-generalized compatible mappings') immediately follows from Theorem 2.2.

Proof Assume that all hypotheses of Theorem 3.1 hold. Consider the mapping $D_{2}: X^{2} \times$ $X^{2} \rightarrow[0, \infty)$ defined, for all $(x, y),(u, v) \in X^{2}$, by

$$
D_{2}((x, y),(u, v))=d(x, u)+d(y, v)
$$

It is well known that $D_{2}$ is a metric on $X^{2}$. Let denote by $T_{F}, T_{H, g}: X^{2} \rightarrow X^{2}$ the mappings given, for all $(x, y) \in X^{2}$, by

$$
T_{F}(x, y)=(F(x, y), F(y, x)) \quad \text { and } \quad T_{H, g}(x, y)=(H(g x, g y), H(g y, g x)) .
$$

Then the following facts hold.

- As $(X, d)$ is complete, then $\left(X^{2}, D_{2}\right)$ is also a complete metric space.

- As $H$ and $g$ are continuous mappings with respect to $d$, then $T_{H, g}$ is also continuous with respect to $D_{2}$.

- As $F$ is a continuous mapping with respect to $d$, then $T_{F}$ is also continuous with respect to $D_{2}$.

- A point $(x, y) \in X^{2}$ is a $g$-coincidence point of $F$ and $H$ (in the sense of Definition 3.1) if, and only if, $(x, y)$ is a coincidence point of $T_{F}$ and $T_{H, g}$. 
- We claim that $T_{F}\left(X^{2}\right) \subseteq T_{H, g}\left(X^{2}\right)$. Indeed, let $(x, y) \in X^{2}$ be arbitrary. By hypothesis, there exist $u, v \in X$ such that $F(x, y)=H(g u, g v)$ and $F(y, x)=H(g v, g u)$. Hence

$$
T_{F}(x, y)=(F(x, y), F(y, x))=(H(g u, g v), H(g v, g u))=T_{H, g}(u, v) \in T_{H, g}\left(X^{2}\right) .
$$

Hence, $T_{F}(x, y) \in T_{H, g}\left(X^{2}\right)$ for all $(x, y) \in X^{2}$, which means that $T_{F}\left(X^{2}\right) \subseteq T_{H, g}\left(X^{2}\right)$.

- We announce that $M$ is $\left(T_{F}, T_{H, g}\right)$-closed. Let $(x, y),(u, v) \in X^{2}$ be such that $\left(T_{H, g}(x, y)\right.$, $\left.T_{H, g}(u, v)\right) \in M$. This is equivalent to saying that

$$
(H(g x, g y), H(g y, g x), H(g u, g v), H(g v, g u)) \in M .
$$

By Definition 3.2, as $M$ is $\left(H_{g}, F\right)$-closed,

$$
\left(T_{F}(x, y), T_{F}(u, v)\right)=(F(x, y), F(y, x), F(u, v), F(v, u)) \in M .
$$

- We show that $M$ is $T_{H, g}$-transitive. Let $(x, y),(u, v),(a, b) \in X^{2}$ be such that $\left(T_{H, g}(x, y)\right.$, $\left.T_{H, g}(u, v)\right) \in M$ and $\left(T_{H, g}(u, v), T_{H, g}(a, b)\right) \in M$. This is equivalent to saying that

$$
\begin{aligned}
& (H(g x, g y), H(g y, g x), H(g u, g v), H(g v, g u)) \in M \quad \text { and } \\
& (H(g u, g v), H(g v, g u), H(g a, g b), H(g b, g a)) \in M .
\end{aligned}
$$

By Definition 3.3, as $M$ is transitive, then

$$
\left(T_{H, g}(x, y), T_{H, g}(a, v)\right)=(H(g x, g y), H(g y, g x), H(g a, g b), H(g b, g a)) \in M .
$$

- Since there exists $\left(x_{0}, y_{0}\right) \in X$ such that

$$
\left(H\left(g x_{0}, g y_{0}\right), H\left(g y_{0}, g x_{0}\right), F\left(x_{0}, y_{0}\right), F\left(y_{0}, x_{0}\right)\right) \in M,
$$

this point trivially verifies $\left(T_{H, g}\left(x_{0}, y_{0}\right), T_{F}\left(x_{0}, y_{0}\right)\right) \in M$. In particular, $M$ is nonempty.

- Let $(x, y),(u, v) \in X^{2}$ be such that $\left(T_{H, g}(x, y), T_{H, g}(u, v)\right) \in M$. This is equivalent to saying that

$$
(H(g x, g y), H(g y, g x), H(g u, g v), H(g v, g u)) \in M .
$$

Using the contractivity condition (2), it follows that

$$
\begin{aligned}
D_{2} & \left(T_{F}(x, y), T_{F}(u, v)\right) \\
& =D_{2}((F(x, y), F(y, x)),(F(u, v), F(v, u))) \\
& =d(F(x, y), F(u, v))+d(F(y, x), F(v, u)) \\
& \leq \varphi(d(H(g x, g y), H(g u, g v))+d(H(g y, g x), H(g v, g u))) \\
& =\varphi\left(D_{2}((H(g x, g y), H(g y, g x)),(H(g u, g v), H(g v, g u)))\right) \\
& =D_{2}\left(T_{H, g}(x, y), T_{H, g}(u, v)\right) .
\end{aligned}
$$

Hence, (1) holds for $T_{F}$ and $T_{H, g}$ using $M \subseteq\left(X^{2}\right)^{2}=X^{4}$. 
- Let us show that $T_{F}$ and $T_{H, g}$ are $(O, M)$-compatible in $\left(X^{2}, D_{2}\right)$ in the sense of Definition 2.6. Let $\left\{\left(x_{n}, y_{n}\right)\right\} \subseteq X^{2}$ be a sequence such that $\left(T_{H, g}\left(x_{n}, y_{n}\right), T_{H, g}\left(x_{n+1}, y_{n+1}\right)\right) \in M$ for all $n \geq 0$ and

$$
\lim _{n \rightarrow \infty} T_{F}\left(x_{n}, y_{n}\right)=\lim _{n \rightarrow \infty} T_{H, g}\left(x_{n}, y_{n}\right)=\left(t_{1}, t_{2}\right) \in X^{2} .
$$

Therefore

$$
\left\{\left(F\left(x_{n}, y_{n}\right), F\left(y_{n}, x_{n}\right)\right)\right\} \stackrel{D_{2}}{\longrightarrow}\left(t_{1}, t_{2}\right) \quad \text { and } \quad\left\{\left(H\left(g x_{n}, g y_{n}\right), H\left(g y_{n}, g x_{n}\right)\right)\right\} \stackrel{D_{2}}{\longrightarrow}\left(t_{1}, t_{2}\right)
$$

As a consequence,

$$
\begin{aligned}
& \left\{F\left(x_{n}, y_{n}\right)\right\} \stackrel{d}{\longrightarrow} t_{1}, \quad\left\{F\left(y_{n}, x_{n}\right)\right\} \stackrel{d}{\rightarrow} t_{2}, \\
& \left\{H\left(g x_{n}, g y_{n}\right)\right\} \stackrel{d}{\longrightarrow} t_{1} \text { and } \quad\left\{H\left(g y_{n}, g x_{n}\right)\right\} \stackrel{d}{\longrightarrow} t_{2} .
\end{aligned}
$$

Since the pair $\{F, H\}$ is $g$-generalized compatible, Definition 3.4 implies that

$$
\left\{\begin{array}{l}
d\left(F\left(H\left(g x_{n}, g y_{n}\right), H\left(g y_{n}, g x_{n}\right)\right), H\left(g F\left(x_{n}, y_{n}\right), g F\left(y_{n}, x_{n}\right)\right)\right) \rightarrow 0 \text { as } n \rightarrow \infty \\
d\left(F\left(H\left(g y_{n}, g x_{n}\right), H\left(g x_{n}, g y_{n}\right)\right), H\left(g F\left(y_{n}, x_{n}\right), g F\left(x_{n}, y_{n}\right)\right)\right) \rightarrow 0 \text { as } n \rightarrow \infty
\end{array}\right.
$$

In particular, the sequence $\left\{D_{2}\left(T_{H, g} T_{F}\left(x_{n}, y_{n}\right), T_{F} T_{H, g}\left(x_{n}, y_{n}\right)\right)\right\}$, which takes the sum of the previous values because, for all $n \in \mathbb{N}$,

$$
\begin{aligned}
D_{2}( & \left.T_{H, g} T_{F}\left(x_{n}, y_{n}\right), T_{F} T_{H, g}\left(x_{n}, y_{n}\right)\right) \\
= & D_{2}\left(T_{H, g}\left(F\left(x_{n}, y_{n}\right), F\left(y_{n}, x_{n}\right)\right), T_{F}\left(H\left(g x_{n}, g y_{n}\right), H\left(g y_{n}, g x_{n}\right)\right)\right) \\
= & D_{2}\left(\left(H\left(g F\left(x_{n}, y_{n}\right), g F\left(y_{n}, x_{n}\right)\right), H\left(g F\left(y_{n}, x_{n}\right), g F\left(x_{n}, y_{n}\right)\right)\right),\right. \\
& \left.\left(\left(F\left(H\left(g x_{n}, g y_{n}\right), H\left(g y_{n}, g x_{n}\right)\right), F\left(H\left(g y_{n}, g x_{n}\right), H\left(g x_{n}, g y_{n}\right)\right)\right)\right)\right) \\
= & d\left(F\left(H\left(g x_{n}, g y_{n}\right), H\left(g y_{n}, g x_{n}\right)\right), H\left(g F\left(x_{n}, y_{n}\right), g F\left(y_{n}, x_{n}\right)\right)\right)+ \\
& +d\left(F\left(H\left(g y_{n}, g x_{n}\right), H\left(g x_{n}, g y_{n}\right)\right), H\left(g F\left(y_{n}, x_{n}\right), g F\left(x_{n}, y_{n}\right)\right)\right),
\end{aligned}
$$

converges to zero. Hence, $T_{F}$ and $T_{H, g}$ are $(O, M)$-compatible in $\left(X^{2}, D_{2}\right)$ in the sense of Definition 2.6.

As a consequence of the previous facts, by using item $\left(\mathrm{a}^{\prime}\right)$ of Theorem 2.2 applied to $T_{F}$ and $T_{H, g}$ in $\left(X^{2}, D_{2}\right)$ and $M \subseteq X^{4}=\left(X^{2}\right)^{2}$, we conclude that $T_{F}$ and $T_{H, g}$ have, at least, a coincidence point, which is a $g$-coincidence point of $F$ and $H$.

In the following result, the continuity of $F$ is not assumed.

Theorem 3.3 (Nan and Charoensawan [13, Theorem 3.2]) Let $(X, d)$ be a complete metric space and $M$ be a nonempty subset of $X^{4}$. Assume that $g: X \rightarrow X$ is continuous and $F, H: X \times X \rightarrow X$ are two generalized compatible mappings such that $H$ is continuous, and for any $x, y \in X$, there exist $u, v \in X$ such that $F(x, y)=H(g u, g v)$ and $F(y, x)=H(g v, g u)$. 
Suppose that there exists $\varphi \in \Phi$ such that the following holds:

$$
\begin{aligned}
& d(F(x, y), F(u, v))+d(F(y, x), F(v, u)) \\
& \quad \leq \varphi(d(H(g x, g y), H(g u, g v))+d(H(g y, g x), H(g v, g u)))
\end{aligned}
$$

for all $x, y, u, v \in X$ with $(H(g x, g y), H(g y, g x), H(g u, g v), H(g v, g u)) \in M$.

Also suppose that $(g(X), d)$ is a complete metric space, $H\left(X^{2}\right) \subseteq g(X)$ and any two sequences $\left\{x_{n}\right\}$ and $\left\{y_{n}\right\}$ with $\left(x_{n}, y_{n}, x_{n+1}, y_{n+1}\right) \in M$ and $\left\{H\left(x_{n}, y_{n}\right)\right\} \rightarrow H(x, y),\left\{H\left(y_{n}, x_{n}\right)\right\} \rightarrow$ $H(y, x)$ for all $n \geq 1$ implies

$$
\left(H\left(x_{n}, y_{n}\right), H\left(y_{n}, x_{n}\right), H(x, y), H(y, x)\right) \in M
$$

for all $n \geq 1$. If there exist $x_{0}, y_{0} \in X$ such that

$$
\left(H\left(g x_{0}, g y_{0}\right), H\left(g y_{0}, g x_{0}\right), F\left(x_{0}, y_{0}\right), F\left(y_{0}, x_{0}\right)\right) \in M
$$

and $M$ is $\left(H_{g}, F\right)$-closed, then there exists $(x, y) \in X \times X$ such that $H(g x, g y)=F(x, y)$ and $H(g y, g x)=F(y, x)$, that is, $F$ and $H$ have a coupled $g$-coincidence point .

The previous statement has the same mistakes as we have pointed out about Theorem 3.1. In fact, the authors assumed the $(X, d)$ and $(g(X), d)$ are, at the same time, complete, which is unnecessary. In this case, we can follow, point by point, the proof of Theorem 3.2, but the continuity of $T_{F}$ is not guaranteed because $F$ is not necessarily continuous. Nevertheless, additional mistakes can be found in its proof. We can easily discover them comparing this result with Theorem 2.2.

Before that, let us show a mistake that can be found in some papers, closely related to item (2) of Remark 2.1. When $F$ is not necessarily continuous, it is usual to assume that the metric space is, in some sense, regular (in the previous result, this assumption is condition (3)). In such a case, the existence of a coincidence point can be deduced applying the contractivity condition to the terms of the sequence and the desired limit. In some cases, this is not possible, as in the following example.

Example 3.1 Assume that $X=\{0,1,2\}$ is endowed with the Euclidean metric $d(x, y)=$ $|x-y|$ for all $x, y \in X$, and $T, g: X \rightarrow X$ and $\varphi:[0, \infty) \rightarrow[0, \infty)$ are defined, for all $x \in X$ and all $t \in[0, \infty)$, by

$$
g x=0, \quad T x=\left\{\begin{array}{ll}
1, & \text { if } x=0, \\
0, & \text { if } x \in\{1,2\},
\end{array} \quad \varphi(t)= \begin{cases}1, & \text { if } t=0, \\
0, & \text { if } t>0 .\end{cases}\right.
$$

Then $\varphi \in \Phi$. If we consider $z=0$ and the sequence $\left\{x_{n}\right\}$ given by

$$
x_{n}= \begin{cases}1, & \text { if } n \text { is even, } \\ 2, & \text { if } n \text { is odd }\end{cases}
$$

then, for all $n \in \mathbb{N}$,

$$
d\left(g x_{n+1}, T z\right)=d(0, T 0)=d(0,1)=1=\varphi(0)=\varphi\left(d\left(g x_{n}, g z\right)\right) .
$$


Although $\left\{x_{n}\right\}$ is a Picard sequence of the pair $(T, g)$ (that is, $g x_{n+1}=T x_{n}$ for all $n \in \mathbb{N}$ ) and $\left\{g x_{n}=0\right\} \rightarrow 0=g z$, it is impossible to deduce that $\left\{g x_{n+1}=0\right\} \rightarrow 1=T z$. Hence, the equality $T z=g z$ cannot be guaranteed from the facts that $\left\{g x_{n}\right\} \rightarrow g z$ and $d\left(g x_{n+1}, T z\right) \leq$ $\varphi\left(d\left(g x_{n}, g z\right)\right)$ for all $n \in \mathbb{N}$. Obviously, the triangular inequality $1=d(0,1)=d(g z, T z) \leq$ $d\left(g z, g x_{n+1}\right)+d\left(g x_{n+1}, T z\right)=0+1$ is not strong enough to conclude that $T z=g z$. This is the main reason why the authors introduced in [17] the notion of $(T, g)$-compatibility (see Definition 2.2).

Next, let us show that some hypotheses of Theorem 3.3 are not appropriate. In fact, we claim that, under appropriate conditions, Theorem 3.3 is a consequence of Theorem 2.2. To prove it, we could try to apply item (c) of the last one. But, comparing both results, we observe three important differences.

- To apply Theorem 2.2 , it would be necessary to assume that

$$
T_{H, g}\left(X^{2}\right)=\left\{(H(g x, g y), H(g y, g x)) \in X^{2}: x, y \in X\right\}
$$

is $D_{2}$-complete (recall Remark 2.4). This condition is guaranteed when $H(g(X) \times g(X))$ is $d$ complete in $X$. However, in Theorem 3.3, it is only assumed that $g(X)$ (and $X$ ) is complete. Although $H(g(X) \times g(X)) \subseteq H\left(X^{2}\right) \subseteq g(X)$, we claim that the completeness of $g(X)$ is not an appropriate hypothesis, as we shall see in the next item.

- Let us show that the regularity condition (3) is not well posed. Following the proof of Theorem 3.3 in [13], the authors had proved, in the previous theorem, that the sequences $\left\{H\left(g x_{n+1}, g y_{n+1}\right)=F\left(x_{n}, y_{n}\right)\right\}$ and $\left\{H\left(g y_{n+1}, g x_{n+1}\right)=F\left(y_{n}, x_{n}\right)\right\}$ are $d$-Cauchy in $H\left(X^{2}\right) \subseteq g(X)$. As a consequence, there were $x, y \in X$ such that

$$
\left\{H\left(g x_{n+1}, g y_{n+1}\right)=F\left(x_{n}, y_{n}\right)\right\} \rightarrow g x \quad \text { and } \quad\left\{H\left(g y_{n+1}, g x_{n+1}\right)=F\left(y_{n}, x_{n}\right)\right\} \rightarrow g y \text {. }
$$

As $H$ is continuous, from the $g$-generalized compatibility, it is possible to deduce that

$$
\begin{aligned}
& \lim _{n \rightarrow \infty} H\left(H\left(g x_{n}, g y_{n}\right), H\left(g y_{n}, g x_{n}\right)\right)=H(g x, g y)=\lim _{n \rightarrow \infty} F\left(H\left(g x_{n}, g y_{n}\right), H\left(g y_{n}, g x_{n}\right)\right) \quad \text { and } \\
& \lim _{n \rightarrow \infty} H\left(H\left(g y_{n}, g x_{n}\right), H\left(g x_{n}, g y_{n}\right)\right)=H(g y, g x)=\lim _{n \rightarrow \infty} F\left(H\left(g y_{n}, g x_{n}\right), H\left(g x_{n}, g y_{n}\right)\right) .
\end{aligned}
$$

Using these limits and the fact that

$$
\left(H\left(g x_{n}, g y_{n}\right), H\left(g y_{n}, g x_{n}\right), H\left(g x_{n+1}, g y_{n+1}\right), H\left(g y_{n+1}, g x_{n+1}\right)\right) \in M
$$

for all $n \in \mathbb{N}$, the authors concluded by (3) that

$$
\left(H\left(H\left(g x_{n}, g y_{n}\right), H\left(g y_{n}, g x_{n}\right)\right), H\left(H\left(g y_{n}, g x_{n}\right), H\left(g x_{n}, g y_{n}\right)\right), H(g x, g y), H(g y, g x)\right) \in M
$$

for all $n \in \mathbb{N}$. To apply the contractivity condition (2), there is necessary a point of the form

$$
\left(H\left(g x^{*}, g y^{*}\right), H\left(g y^{*}, g x^{*}\right), H\left(g u^{*}, g v^{*}\right), H\left(g v^{*}, g u^{*}\right)\right) \in M \text {. }
$$


As $H\left(X^{2}\right) \subseteq g(X)$, then there exist $\left\{z_{n}\right\},\left\{\omega_{n}\right\} \subseteq X$ such that

$$
H\left(g x_{n}, g y_{n}\right)=g z_{n} \quad \text { and } \quad H\left(g y_{n}, g x_{n}\right)=g \omega_{n} \quad \text { for all } n \in \mathbb{N} .
$$

Hence, (4) is equivalent to

$$
\left(H\left(g z_{n}, g \omega_{n}\right), H\left(g \omega_{n}, g z_{n}\right), H(g x, g y), H(g y, g x)\right) \in M \quad \text { for all } n \in \mathbb{N} \text {. }
$$

In this case, the contractivity condition (2) yields

$$
\begin{aligned}
& d\left(F\left(z_{n}, \omega_{n}\right), F(x, y)\right)+d\left(F\left(\omega_{n}, z_{n}\right), F(y, x)\right) \\
& \quad \leq \varphi\left(d\left(H\left(g z_{n}, g \omega_{n}\right), H(g x, g y)\right)+d\left(H\left(g \omega_{n}, g z_{n}\right), H(g y, g x)\right)\right) .
\end{aligned}
$$

In the left-hand side of this inequality, there appears $F\left(z_{n}, \omega_{n}\right)$ and $F\left(\omega_{n}, z_{n}\right)$, but the authors wrote an inequality involving

$$
\begin{aligned}
& F\left(g z_{n}, g \omega_{n}\right)=F\left(H\left(g x_{n}, g y_{n}\right), H\left(g y_{n}, g x_{n}\right)\right) \quad \text { and } \\
& F\left(g \omega_{n}, g z_{n}\right)=F\left(H\left(g y_{n}, g x_{n}\right), H\left(g x_{n}, g y_{n}\right)\right),
\end{aligned}
$$

which cannot be obtained from the contractivity condition. This means that the regularity condition (3) is not well posed because it is not compatible with the contractivity condition (and the fact that $H(g(X) \times g(X))$ is not necessarily $d$-complete).

- Furthermore, comparing Theorem 3.3 and Theorem 2.2, we observe that the hypothesis of $(T, g)$-compatibility is omitted. This leads us to the last mistake in the proof given by the authors of Theorem 3.3. As we have just seen, although the contractivity condition is not applicable as follows, on [13, p.12], the authors announced that, for all $n \in \mathbb{N}$,

$$
\begin{aligned}
& d(H(g x, g y), F(x, y))+d(H(g y, g x), F(y, x)) \\
& \leq d\left(H(g x, g y), F\left(H\left(g x_{n}, g y_{n}\right), H\left(g y_{n}, g x_{n}\right)\right)\right) \\
& \quad+d\left(F\left(H\left(g x_{n}, g y_{n}\right), H\left(g y_{n}, g x_{n}\right)\right), F(x, y)\right) \\
& \quad+d\left(H(g y, g x), F\left(H\left(g y_{n}, g x_{n}\right), H\left(g x_{n}, g y_{n}\right)\right)\right) \\
& \quad+d\left(F\left(H\left(g y_{n}, g x_{n}\right), H\left(g x_{n}, g y_{n}\right)\right), F(x, y)\right) \\
& \leq \varphi\left(d\left(H\left(H\left(g x_{n}, g y_{n}\right), H\left(g y_{n}, g x_{n}\right)\right), H(g x, g y)\right)\right. \\
&+d\left(H\left(H\left(g y_{n}, g x_{n}\right), H\left(g x_{n}, g y_{n}\right)\right), H(g y, g x)\right) \\
&+d\left(H(g x, g y), H\left(H\left(g x_{n}, g y_{n}\right), H\left(g y_{n}, g x_{n}\right)\right)\right. \\
&+d\left(H(g y, g x), H\left(H\left(g y_{n}, g x_{n}\right), H\left(g x_{n}, g y_{n}\right)\right)\right) .
\end{aligned}
$$

Clearly, (6) is incorrectly established because the contractivity condition yields terms with two summands in the argument of $\varphi$. In any case, the correct version would be

$$
\begin{aligned}
& d(H(g x, g y), F(x, y))+d(H(g y, g x), F(y, x)) \\
& \quad \leq \varphi\left(d\left(H\left(H\left(g x_{n}, g y_{n}\right), H\left(g y_{n}, g x_{n}\right)\right), H(g x, g y)\right)\right.
\end{aligned}
$$




$$
\begin{aligned}
& \left.+d\left(H\left(H\left(g y_{n}, g x_{n}\right), H\left(g x_{n}, g y_{n}\right)\right), H(g y, g x)\right)\right) \\
& +d\left(H(g x, g y), H\left(H\left(g x_{n}, g y_{n}\right), H\left(g y_{n}, g x_{n}\right)\right)\right. \\
& +d\left(H(g y, g x), H\left(H\left(g y_{n}, g x_{n}\right), H\left(g x_{n}, g y_{n}\right)\right)\right.
\end{aligned}
$$

where the sequence inside the argument of $\varphi$ tends to zero. As $\varphi \in \Phi$, the authors used the fact that $\lim _{t \rightarrow 0^{+}} \varphi(t)=0$ to conclude that $d(H(g x, g y), F(x, y))=d(H(g y, g x), F(y, x))=0$. As we have shown in Example 3.1, this reasoning is not correct. It would be necessary to assume some additional hypothesis (for instance, $\varphi(0)=0$ or a kind of $\left(T_{F}, T_{H, g}\right)$ compatibility) in order to conclude that $d(H(g x, g y), F(x, y))=d(H(g y, g x), F(y, x))=0$.

To overcome the previous drawbacks, it would be convenient to consider the following hypotheses.

(1) $H(g(X) \times g(X))$ is complete in $(X, d)$, which means that $T_{H, g}\left(X^{2}\right)$ is complete in $\left(X^{2}, D_{2}\right)$.

(2) The regularity condition (3) must be replaced by the following one: 'if $\left\{x_{n}\right\},\left\{y_{n}\right\} \subseteq X$ are sequences such that $\left\{x_{n}\right\} \rightarrow x \in X,\left\{y_{n}\right\} \rightarrow y \in X$ and $\left(x_{n}, y_{n}, x_{n+1}, y_{n+1}\right) \in M$ for all $n \in \mathbb{N}$, then $\left(x_{n}, y_{n}, x, y\right) \in M$ for all $n \in \mathbb{N}$. In such a case, $\left(X^{2}, D_{2}, M\right)$ is regular in the sense of Definition 2.4.

(3) A kind of $\left(T_{F}, T_{H, g}\right)$-compatibility is necessary to ensure that the limit as $n \rightarrow \infty$ in (7) is zero. For instance, we propose assuming that if $(x, y, u, v) \in M$ is such that $H(g x, g y)=$ $H(g u, g v)$ and $H(g y, g x)=H(g v, g u)$, then $F(x, y)=F(u, v)$ and $F(y, x)=F(v, u)$. In this case, $M$ is $\left(T_{F}, T_{H, g}\right)$-compatible. This condition can be omitted if we additionally assume that $\varphi(0)=0$ (see, for instance, Corollary 35 in [15]).

Under these new conditions, Theorem 3.3 becomes a consequence of Theorem 2.2.

\section{About some coupled coincidence point theorems in G-metric spaces}

All necessary preliminaries (about quasi-metrics, G-metrics, contractions depending on a subset $\mathcal{M} \subseteq X^{2}$, etc.) of this part can be found in [18]. Let $\Psi$ be the family of functions $\varphi \in \Phi$ such that $\varphi(t)=0$ if, and only if, $t=0$ (notice that $\Psi$ and $\Phi$ were employed in [18] using the contrary notation). The following result shows a simple way to consider quasimetrics from $G$-metrics.

Lemma 4.1 (Agarwal et al. [19]) Let $(X, G)$ be a G-metric space and let us define $q_{G}, q_{G}^{\prime}$ : $X^{2} \rightarrow[0, \infty)$ by

$$
q_{G}(x, y)=G(x, y, y) \quad \text { and } \quad q_{G}^{\prime}(x, y)=G(x, x, y) \quad \text { for all } x, y \in X .
$$

Then the following properties hold.

(1) $q_{G}$ and $q_{G}^{\prime}$ are quasi-metrics on $X$. Moreover,

$$
q_{G}^{\prime}(x, y) \leq 2 q_{G}(x, y) \leq 4 q_{G}^{\prime}(x, y) \quad \text { for all } x, y \in X
$$

(2) In $\left(X, q_{G}\right)$ and in $\left(X, q_{G}^{\prime}\right)$, a sequence is right-convergent (respectively, left-convergent) if and only if it is convergent. In such a case, its right-limit, its left-limit and its limit coincide.

(3) In $\left(X, q_{G}\right)$ and in $\left(X, q_{G}^{\prime}\right)$, a sequence is right-Cauchy (respectively, left-Cauchy) if and only if it is Cauchy. 
(4) In $\left(X, q_{G}\right)$ and in $\left(X, q_{G}^{\prime}\right)$, every right-convergent (respectively, left-convergent) sequence has a unique right-limit (respectively, left-limit).

(5) If $\left\{x_{n}\right\} \subseteq X$ and $x \in X$, then $\left\{x_{n}\right\} \stackrel{G}{\longrightarrow} x \Longleftrightarrow\left\{x_{n}\right\} \stackrel{q_{G}}{\longrightarrow} x \Longleftrightarrow\left\{x_{n}\right\} \stackrel{q_{G}^{\prime}}{\longrightarrow} x$.

(6) If $\left\{x_{n}\right\} \subseteq X$, then $\left\{x_{n}\right\}$ is G-Cauchy $\Longleftrightarrow\left\{x_{n}\right\}$ is $q_{G}$-Cauchy $\Longleftrightarrow\left\{x_{n}\right\}$ is $q_{G}^{\prime}$-Cauchy.

(7) $(X, G)$ is complete $\Longleftrightarrow\left(X, q_{G}\right)$ is complete $\Longleftrightarrow\left(X, q_{G}^{\prime}\right)$ is complete.

Definition 4.1 Let $(X, q)$ be a quasi-metric space and let $\mathcal{M}$ be a nonempty subset of $X^{2}$. Two mappings $T, g: X \rightarrow X$ are said to be $(O, \mathcal{M})$-compatible if

$$
\lim _{m \rightarrow \infty} q\left(g T x_{m}, \operatorname{Tg} x_{m}\right)=0 \text { and } \lim _{m \rightarrow \infty} q\left(\operatorname{Tg} x_{m}, g T x_{m}\right)=0
$$

provided that $\left\{x_{m}\right\}$ is a sequence in $X$ such that $\left(g x_{n}, g x_{m}\right) \in \mathcal{M}$ for all $n<m$ and

$$
\lim _{m \rightarrow \infty} T x_{m}=\lim _{m \rightarrow \infty} g x_{m} \in X
$$

Similarly, $T$ and $g$ are said to be $\left(O^{\prime}, \mathcal{M}\right)$-compatible if

$$
\lim _{m \rightarrow \infty} q\left(g T x_{m}, \operatorname{Tg} x_{m}\right)=0 \quad \text { or } \quad \lim _{m \rightarrow \infty} q\left(\operatorname{Tg} x_{m}, g T x_{m}\right)=0
$$

provided that $\left\{x_{m}\right\}$ is a sequence in $X$ such that $\left(g x_{n}, g x_{m}\right) \in \mathcal{M}$ for all $n<m$ and

$$
\lim _{m \rightarrow \infty} T x_{m}=\lim _{m \rightarrow \infty} g x_{m} \in X
$$

Clearly, if $T$ and $g$ are commuting, then they are both $(O, \mathcal{M})$-compatible or $\left(O^{\prime}, \mathcal{M}\right)$ compatible. The following notion also extends the regularity of an ordered metric space.

Definition 4.2 Let $(X, q)$ be a quasi-metric space and let $A \subseteq X$ and $\mathcal{M} \subseteq X^{2}$ be two nonempty subsets. We say that $(A, q, \mathcal{M})$ is regular (or $A$ is $(q, \mathcal{M})$-regular) if we have $\left(x_{n}, u\right) \in \mathcal{M}$ for all $n$ provided that $\left\{x_{n}\right\}$ is a $q$-convergent sequence on $A, u \in A$ is its $q$ limit and $\left(x_{n}, x_{m}\right) \in \mathcal{M}$ for all $n<m$.

Definition 4.3 Let $(X, q)$ be a quasi-metric space, let $T, g: X \rightarrow X$ be two mappings and let $\mathcal{M} \subseteq X^{2}$ be a nonempty subset of $X^{2}$. We say that $T$ is a $(g, \mathcal{M}, \Psi)$-contraction of the second kind if there exists $\varphi \in \Psi$ such that

$$
q(T x, T y) \leq \varphi(q(g x, g y))
$$

for all $x, y \in X$ such that $(g x, g y) \in \mathcal{M}$. If $\varphi \in \Phi$, we say that $T$ is a $(g, \mathcal{M}, \Phi)$-contraction of the second kind.

Notice that condition (8) is not symmetric on $x$ and $y$ because $(g x, g y) \in \mathcal{M}$ does not imply $(g y, g x) \in \mathcal{M}$. In order to compensate this absence of symmetry, we will suppose an additional condition on the ambient space.

Definition 4.4 We say that a quasi-metric space $(X, q)$ is:

- right-Cauchy if every right-Cauchy sequence in $(X, q)$ is, in fact, a Cauchy sequence in $(X, q)$; 
- left-Cauchy if every left-Cauchy sequence in $(X, q)$ is, in fact, a Cauchy sequence in $(X, q)$;

- right-convergent if every right-convergent sequence in $(X, q)$ is, in fact, a convergent sequence in $(X, q)$;

- left-convergent if every left-convergent sequence in $(X, q)$ is, in fact, a convergent sequence in $(X, q)$.

Theorem 4.1 (Roldán-López-de-Hierro et al. [18, Theorem 3.3]) Let $(X, q)$ be a rightCauchy quasi-metric space, let $T, g: X \rightarrow X$ be two mappings and let $\mathcal{M}$ be a nonempty subset of $X^{2}$. Suppose that the following conditions are fulfilled.

(A) There exists a $(T, g, \mathcal{M})$-Picard sequence on $X$.

(B) $T$ is a $(g, \mathcal{M}, \Psi)$-contraction of the second kind.

Also assume that, at least, one of the following conditions holds.

(a) $X($ or $g(X)$ or $T(X))$ is $q$-complete, $T$ and $g$ are $\mathcal{M}$-continuous and the pair $(T, g)$ is $\left(O^{\prime}, \mathcal{M}\right)$-compatible;

(b) $X($ or $g(X)$ or $T(X))$ is $q$-complete and $T$ and $g$ are $\mathcal{M}$-continuous and commuting;

(c) $(g(X), q)$ is complete and right-convergent, and $X($ or $g(X))$ is $(q, \mathcal{M})$-regular;

(d) $(X, q)$ is complete and right-convergent, $g(X)$ is closed and $X($ or $g(X))$ is $(q, \mathcal{M})$-regular

(e) $(X, q)$ is complete and right-convergent, $g$ is $\mathcal{M}$-continuous, $\mathcal{M}$ is $g$-closed, the pair $(T, g)$ is $(O, \mathcal{M})$-compatible and $X$ is $(q, \mathcal{M})$-regular.

Then $T$ and $g$ have, at least, a coincidence point.

Immediately, it was pointed out that the previous result also holds if we replace condition (A) by one of the following stronger hypotheses:

$\left(\mathrm{A}^{\prime}\right) \quad T(X) \subseteq g(X), \mathcal{M}$ is $g$-transitive and $(T, g)$-closed, and there exists $x_{0} \in X$ such that $\left(g x_{0}, T x_{0}\right) \in \mathcal{M}$.

$\left(\mathrm{A}^{\prime \prime}\right) \mathcal{M}$ is $g$-transitive and $(T, g)$-closed, and there exists a $(T, g)$-Picard sequence $\left\{x_{n}\right\}_{n \geq 0}$ such that $\left(g x_{0}, T x_{0}\right) \in \mathcal{M}$.

The $\mathcal{M}$-continuity of the mappings can be replaced by continuity. The previous result was extended to the more general case in which $\varphi \in \Phi$ as follows.

Remark 4.1 Notice that the hypothesis 'there exists $x_{0} \in X$ such that $\left(g x_{0}, T x_{0}\right) \in \mathcal{M}$ ' was omitted in condition $\left(\mathrm{A}^{\prime}\right)$ by mistake in [18].

Theorem 4.2 If we additionally assume that $\mathcal{M}$ is $(T, g)$-compatible, then Theorem 4.1 also holds even if $T$ is a $(g, \mathcal{M}, \Phi)$-contraction of the second kind.

Remark 4.2 As we pointed out in the previous section, throughout the proof of Theorem 4.1 in [18], the $(T, g)$-compatibility of $\mathcal{M}$ was only used under assumptions (c), (d), and (e), when $X$ (or $g(X))$ is $(q, \mathcal{M})$-regular. However, when $T$ and $g$ are continuous (or $\mathcal{M}$-continuous), it is not necessary.

In [14], the authors introduced the following notions in which $F, H: X \times X \rightarrow X$ are two arbitrary mappings, and they proved the following theorem. 
Definition 4.5 An element $(x, y) \in X^{2}$ is called a coupled coincidence point of the mappings $F$ and $H$ if $F(x, y)=H(x, y)$ and $F(y, x)=H(y, x)$.

Definition 4.6 Let $M$ be a subset of $X^{6}$. We say that $M$ is an $(H, F)$-closed subset of $X^{6}$ if, for all $x, y, z, u, v, w \in X$,

$$
\begin{aligned}
& (H(x, u), H(u, x), H(y, v), H(v, y), H(z, w), H(w, z)) \in M \\
& \quad \Rightarrow \quad(F(x, u), F(u, x), F(y, v), F(v, y), F(z, w), F(w, z)) \in M .
\end{aligned}
$$

Definition 4.7 Let $M$ be a subset of $X^{6}$. We say that $M$ satisfies the transitive property if and only if for all $x, y, z, u, v, w, a, b, c, d \in X$,

$$
\begin{aligned}
& (H(x, u), H(u, x), H(y, v), H(v, y), H(a, b), H(b, a)) \in M \quad \text { and } \\
& (H(a, b), H(b, a), H(c, d), H(d, c), H(z, w), H(w, z)) \in M \\
& \quad \Rightarrow \quad(H(x, u), H(u, x), H(y, v), H(v, y), H(z, w), H(w, z)) \in M .
\end{aligned}
$$

Definition 4.8 We say that the pair $\{F, H\}$ is generalized compatible if $\left\{x_{n}\right\}$ and $\left\{y_{n}\right\}$ are sequences in $X$ such that for some $x, y \in X$

$$
\lim _{n \rightarrow \infty} H\left(x_{n}, y_{n}\right)=\lim _{n \rightarrow \infty} F\left(x_{n}, y_{n}\right)=x \quad \text { and } \quad \lim _{n \rightarrow \infty} H\left(y_{n}, x_{n}\right)=\lim _{n \rightarrow \infty} F\left(y_{n}, x_{n}\right)=y
$$

imply

$$
\begin{aligned}
& \lim _{n \rightarrow \infty} F\left(H\left(x_{n}, y_{n}\right), H\left(y_{n}, x_{n}\right)\right)=\lim _{n \rightarrow \infty} H\left(F\left(x_{n}, y_{n}\right), F\left(y_{n}, x_{n}\right)\right) \quad \text { and } \\
& \lim _{n \rightarrow \infty} F\left(H\left(y_{n}, x_{n}\right), H\left(x_{n}, y_{n}\right)\right)=\lim _{n \rightarrow \infty} H\left(F\left(y_{n}, x_{n}\right), F\left(x_{n}, y_{n}\right)\right) .
\end{aligned}
$$

Notice that, although the authors did not remark it in [14], the previous definition needs a metric structure, maybe in a metric space, in a quasi-metric space or in a $G$-metric space. The first main result in [14] is the following one.

Theorem 4.3 (Nan and Charoensawan [14, Theorem 3.1]) Let $(X, \preceq)$ be a partially ordered set and $G$ be a G-metric on $X$ such that $(X, G)$ is a complete G-metric space and $M$ be a nonempty subset of $X^{6}$. Assume that $F, H: X \times X \rightarrow X$ are two generalized compatible mappings such that $H$ is continuous and for any $x, y \in X$, there exist $u, v \in X$ such that $F(x, y)=H(u, v)$ and $F(y, x)=H(v, u)$. Suppose that there exists $\varphi \in \Phi$ such that the following holds:

$$
\begin{aligned}
& G(F(x, u), F(y, v), F(z, w))+G(F(u, x), F(v, y), F(w, z)) \\
& \quad \leq \varphi(G(H(x, u), H(y, v), H(z, w))+G(H(u, x), H(v, y), H(w, z)))
\end{aligned}
$$

for all $x, y, z, u, v, w \in X$ with $(H(x, u), H(u, x), H(y, v), H(v, y), H(z, w), H(w, z)) \in M$.

Suppose also that either:

(a) F is continuous; 
(b) for any two sequences $\left\{x_{n}\right\}$ and $\left\{y_{n}\right\}$ with for all $n \geq 1$

$$
\begin{aligned}
& \left(x_{n+1}, y_{n+1}, x_{n+1}, y_{n+1}, x_{n}, y_{n}\right) \in M \text { and } \\
& \left\{H\left(x_{n}, y_{n}\right)\right\} \rightarrow H(x, y), \quad\left\{H\left(y_{n}, x_{n}\right)\right\} \rightarrow H(y, x) \\
& \quad \text { implies } \\
& \left(H\left(x_{n}, y_{n}\right), H\left(y_{n}, x_{n}\right), H(x, y), H(y, x), H(x, y), H(y, x)\right) \in M .
\end{aligned}
$$

If there exists $\left(x_{0}, y_{0}\right) \in X \times X$ such that

$$
\left(F\left(x_{0}, y_{0}\right), F\left(y_{0}, x_{0}\right), F\left(x_{0}, y_{0}\right), F\left(y_{0}, x_{0}\right), H\left(x_{0}, y_{0}\right), H\left(y_{0}, x_{0}\right)\right) \in M
$$

and $M$ is $(H, F)$-closed, then there exists $(x, y) \in X \times X$ such that $H(x, y)=F(x, y)$ and $H(y, x)=F(y, x)$, that is, $F$ and $H$ have a coupled coincidence point.

At first sight, the reader can easily observe the following mistakes.

- The partial order $\preceq$ is a superfluous hypothesis.

- The authors did not assume the transitive property in the statement although they used it throughout the proof.

Following the techniques we have shown in the first part of the manuscript, we may deduce the following statement.

Theorem 4.4 Under condition (a), Theorem 4.3 immediately follows from Theorem 4.2.

Proof Suppose that all hypotheses of Theorem 4.3 hold. Given the G-metric G, let us define the mapping $q_{G}: X^{2} \times X^{2} \rightarrow[0, \infty)$, for all $(x, y),(u, v) \in X^{2}$, by

$$
q_{G}((x, y),(u, v))=G(x, u, u)+G(y, v, v) .
$$

Following the same arguments of Lemma 4.1, it is not difficult to show that $q_{G}$ is a quasimetric in $X^{2}$.

One of the key objectives of this proof is to show that, in Theorem 4.3, the middle variables of $M$ are not necessary. Indeed, given a nonempty subset $M \subseteq X^{6}$, let us define

$$
M^{\prime}=\left\{(x, u, y, v) \in X^{4}:(y, v, y, v, x, u) \in M\right\} .
$$

Notice that $M^{\prime}$ is a subset of $X^{4}=X^{2} \times X^{2}$.

Next, let us define the mappings $T_{F}, T_{H}: X^{2} \rightarrow X^{2}$, for all $(x, y) \in X^{2}$, by

$$
T_{F}(x, y)=(F(x, y), F(y, x)) \quad \text { and } \quad T_{H}(x, y)=(H(x, y), H(y, x)) .
$$

Then the following facts hold.

- As $(X, G)$ is a complete $G$-metric space, then $\left(X^{2}, q_{G}\right)$ is a complete quasi-metric space.

- As $q_{G}$ comes from a $G$-metric, then $\left(X^{2}, q_{G}\right)$ is a right-Cauchy quasi-metric space.

- As $F$ (respectively, $H$ ) is a continuous mapping with respect to $G$, then $T_{F}$ (respectively, $\left.T_{H}\right)$ is also continuous with respect to $q_{G}$. 
- A point $(x, y) \in X^{2}$ is a coupled coincidence point of $F$ and $H$ (in the sense of Definition 4.5) if, and only if, $(x, y)$ is a coincidence point of $T_{F}$ and $T_{H}$.

- Since there exists $\left(x_{0}, y_{0}\right) \in X$ such that

$$
\left(F\left(x_{0}, y_{0}\right), F\left(y_{0}, x_{0}\right), F\left(x_{0}, y_{0}\right), F\left(y_{0}, x_{0}\right), H\left(x_{0}, y_{0}\right), H\left(y_{0}, x_{0}\right)\right) \in M
$$

we have

$$
\left(T_{H}\left(x_{0}, y_{0}\right), T_{F}\left(x_{0}, y_{0}\right)\right)=\left(H\left(x_{0}, y_{0}\right), H\left(y_{0}, x_{0}\right), F\left(x_{0}, y_{0}\right), F\left(y_{0}, x_{0}\right)\right) \in M^{\prime}
$$

In particular, $M^{\prime}$ is nonempty.

- We claim that $T_{F}\left(X^{2}\right) \subseteq T_{H}\left(X^{2}\right)$. Indeed, let $(x, y) \in X^{2}$ be arbitrary. By hypothesis, there exist $u, v \in X$ such that $F(x, y)=H(u, v)$ and $F(y, x)=H(v, u)$. Hence

$$
T_{F}(x, y)=(F(x, y), F(y, x))=(H(u, v), H(v, u))=T_{H}(u, v) \in T_{H}\left(X^{2}\right) .
$$

Therefore, $T_{F}(x, y) \in T_{H}\left(X^{2}\right)$ for all $(x, y) \in X^{2}$, which means that $T_{F}\left(X^{2}\right) \subseteq T_{H}\left(X^{2}\right)$.

- We announce that $M^{\prime}$ is $\left(T_{F}, T_{H}\right)$-closed. Let $(x, y),(u, v) \in X^{2}$ be such that $\left(T_{H}(x, y)\right.$, $\left.T_{H}(u, v)\right) \in M^{\prime}$. This is equivalent to saying that

$$
(H(u, v), H(v, u), H(u, v), H(v, u), H(x, y), H(y, x)) \in M .
$$

By Definition 4.6, as $M$ is $(H, F)$-closed,

$$
(F(u, v), F(v, u), F(u, v), F(v, u), F(x, y), F(y, x)) \in M .
$$

which means that $\left(T_{F}(x, y), T_{F}(u, v)\right)=(F(x, y), F(y, x), F(u, v), F(v, u)) \in M^{\prime}$.

- We show that $M^{\prime}$ is $T_{H}$-transitive. Let $(x, y),(u, v),(a, b) \in X^{2}$ be such that $\left(T_{H}(x, y)\right.$, $\left.T_{H}(u, v)\right) \in M^{\prime}$ and $\left(T_{H}(u, v), T_{H}(a, b)\right) \in M^{\prime}$. This is equivalent to saying that

$$
\begin{aligned}
& (H(u, v), H(v, u), H(u, v), H(v, u), H(x, y), H(y, x)) \in M \quad \text { and } \\
& (H(a, b), H(b, a), H(a, b), H(b, a), H(u, v), H(v, u)) \in M .
\end{aligned}
$$

By Definition 4.7, as $M$ is transitive, we have

$$
(H(a, b), H(b, a), H(a, b), H(b, a), H(x, y), H(y, x)) \in M,
$$

which implies that

$$
\left(T_{H}(x, y), T_{H}(a, b)\right)=(H(x, y), H(y, x), H(a, b), H(b, a)) \in M^{\prime} .
$$

- We claim that the mappings $T_{F}$ and $T_{H}$ are $\left(O^{\prime}, M^{\prime}\right)$-compatible (and also $\left(O, M^{\prime}\right)$ compatible) in $\left(X^{2}, q_{G}\right)$ in the sense of Definition 4.1. Assume that $\left\{\left(x_{n}, y_{n}\right)\right\} \subseteq X^{2}$ is a sequence such that

$$
\left(T_{H}\left(x_{n}, y_{n}\right), T_{H}\left(x_{m}, y_{m}\right)\right) \in M^{\prime} \quad \text { for all } n<m, \quad \text { and }
$$




$$
\left\{T_{F}\left(x_{n}, y_{n}\right)\right\} \stackrel{q_{G}}{\longrightarrow}(z, \omega) \in X^{2}, \quad\left\{T_{H}\left(x_{n}, y_{n}\right)\right\} \stackrel{q_{G}}{\longrightarrow}(z, \omega) \in X^{2} .
$$

This means that

$$
\begin{array}{ll}
\left\{F\left(x_{n}, y_{n}\right)\right\} \stackrel{G}{\rightarrow} z, & \left\{F\left(y_{n}, x_{n}\right)\right\} \stackrel{G}{\rightarrow} \omega \\
\left\{H\left(x_{n}, y_{n}\right)\right\} \stackrel{G}{\longrightarrow} z, & \left\{H\left(y_{n}, x_{n}\right)\right\} \stackrel{G}{\longrightarrow} \omega .
\end{array}
$$

As $F$ and $H$ are two generalized compatible mappings (in the sense of Definition 4.8), the following limits in $(X, G)$ are the same:

$$
\begin{aligned}
& \lim _{n \rightarrow \infty} F\left(H\left(x_{n}, y_{n}\right), H\left(y_{n}, x_{n}\right)\right)=\lim _{n \rightarrow \infty} H\left(F\left(x_{n}, y_{n}\right), F\left(y_{n}, x_{n}\right)\right) \quad \text { and } \\
& \lim _{n \rightarrow \infty} F\left(H\left(y_{n}, x_{n}\right), H\left(x_{n}, y_{n}\right)\right)=\lim _{n \rightarrow \infty} H\left(F\left(y_{n}, x_{n}\right), F\left(x_{n}, y_{n}\right)\right) .
\end{aligned}
$$

As a consequence,

$$
\begin{aligned}
\lim _{n \rightarrow \infty} & q_{G}\left(T_{H} T_{F}\left(x_{n}, y_{n}\right), T_{F} T_{H}\left(x_{n}, y_{n}\right)\right) \\
= & \lim _{n \rightarrow \infty} q_{G}\left(T_{H}\left(F\left(x_{n}, y_{n}\right), F\left(y_{n}, x_{n}\right)\right), T_{F}\left(H\left(x_{n}, y_{n}\right), H\left(y_{n}, x_{n}\right)\right)\right) \\
= & \lim _{n \rightarrow \infty} q_{G}\left(\left(H\left(F\left(x_{n}, y_{n}\right), F\left(y_{n}, x_{n}\right)\right), H\left(F\left(y_{n}, x_{n}\right), F\left(x_{n}, y_{n}\right)\right)\right),\right. \\
& \left.\left(F\left(H\left(x_{n}, y_{n}\right), H\left(y_{n}, x_{n}\right)\right), F\left(H\left(y_{n}, x_{n}\right), H\left(x_{n}, y_{n}\right)\right)\right)\right) \\
= & \lim _{n \rightarrow \infty}\left[G\left(H\left(F\left(x_{n}, y_{n}\right), F\left(y_{n}, x_{n}\right)\right), F\left(H\left(x_{n}, y_{n}\right), H\left(y_{n}, x_{n}\right)\right), F\left(H\left(x_{n}, y_{n}\right), H\left(y_{n}, x_{n}\right)\right)\right)\right. \\
& \left.+G\left(H\left(F\left(y_{n}, x_{n}\right), F\left(x_{n}, y_{n}\right)\right), F\left(H\left(y_{n}, x_{n}\right), H\left(x_{n}, y_{n}\right)\right), F\left(H\left(y_{n}, x_{n}\right), H\left(x_{n}, y_{n}\right)\right)\right)\right] \\
= & 0 .
\end{aligned}
$$

Hence, the mappings $T_{F}$ and $T_{H}$ are $\left(O^{\prime}, M^{\prime}\right)$-compatible in $\left(X^{2}, q_{G}\right)$.

- Let $(x, y),(u, v) \in X^{2}$ be such that $\left(T_{H}(x, y), T_{H}(u, v)\right) \in M^{\prime}$. This is equivalent to saying that

$$
(H(u, v), H(v, u), H(u, v), H(v, u), H(x, y), H(y, x)) \in M .
$$

Using the contractivity condition (9), we deduce that

$$
\begin{aligned}
q_{G} & \left(T_{F}(x, y), T_{F}(u, v)\right) \\
& =q_{G}((F(x, y), F(y, x)),(F(u, v), F(v, u))) \\
& =G(F(x, y), F(u, v), F(u, v))+G(F(y, x), F(v, u), F(v, u)) \\
& \leq \varphi(G(H(x, y), H(u, v), H(u, v))+G(H(y, x), H(v, u), H(v, u))) \\
& =\varphi\left(q_{G}((H(x, y), H(y, x)),(H(u, v), H(v, u)))\right) \\
& =\varphi\left(q_{G}\left(T_{H}(x, y), T_{H}(u, v)\right)\right) .
\end{aligned}
$$

This means that $T_{F}$ is a $\left(T_{H}, M^{\prime}, \Phi\right)$-contraction of the second kind in $\left(X^{2}, q_{G}\right)$. 
As a consequence of Theorem 4.2, $T_{F}$ and $T_{H}$ have, at least, a coincidence point, which is a coupled coincidence point of $F$ and $H$.

With respect to the case in which $F$ is not necessarily continuous and we assume the regularity condition (hypothesis (b) of Theorem 4.3), we can now repeat the same commentaries that we gave in the previous section.

\section{Competing interests}

The authors declare that they have no competing interests.

\section{Authors' contributions}

All authors contributed equally and significantly in writing this article. All authors read and approved the final manuscript.

\section{Author details}

${ }^{1}$ Department of Mathematics, Atilim University, İncek, Ankara, 06836, Turkey. ${ }^{2}$ University of Jaén, Campus las Lagunillas s/n, Jaén, 23071, Spain. ${ }^{3}$ Department of Mathematics, King Abdulaziz University, P.O. Box 80203, Jeddah, 21589, Saudi Arabia.

\section{Acknowledgements}

This article was funded by the Deanship of Scientific Research (DSR), King Abdulaziz University, Jeddah. N Shahzad acknowledges with thanks DSR for financial support. A-F Roldán-López-de-Hierro has been partially supported by Junta de Andalucía by project FQM-268 of the Andalusian CICYE.

Received: 24 October 2014 Accepted: 13 January 2015 Published online: 12 February 2015

\section{References}

1. Turinici, M: Abstract comparison principle and multivariable Gronwall-Bellman inequalities. J. Math. Anal. Appl. 117, 100-127 (1986)

2. Ran, ACM, Reurings, MCB: A fixed point theorem in partially ordered sets and some applications to matrix equations. Proc. Am. Math. Soc. 132, 1435-1443 (2004)

3. Nieto, JJ, Rodríguez-López, R: Contractive mapping theorem in partially ordered sets and applications to ordinary differential equations. Order 22, 223-239 (2005)

4. Kadelburg, Z, Nashine, HK, Radenović, S: Common coupled fixed point results in partially ordered G-metric spaces. Bull. Math. Anal. Appl. 4(2), 51-63 (2012)

5. Agarwal, RP, Kadelburg, Z, Radenović, S: On coupled fixed point results in asymmetric G-metric spaces. J. Inequal. Appl. 2013, Article ID 528 (2013)

6. Radenović, S: Remarks on some recent coupled coincidence point results in symmetric G-metric spaces. J. Oper. 2013, Article ID 290525 (2013)

7. Radenović, S: A note on tripled coincidence and tripled common fixed point theorems in partially ordered metric spaces. Appl. Math. Comput. 236, 367-372 (2014)

8. Radenović, S: Remarks on some coupled coincidence point results in partially ordered metric spaces. Arab J. Math. Sci. 20(1), 29-39 (2014)

9. Roldán, A, Martínez-Moreno, J, Roldán, C, Karapınar, E: Some remarks on multidimensional fixed point theorems. Fixed Point Theory 15(2), 545-558 (2014)

10. Alsulami, HH, Roldán-López-de-Hierro, AF, Karapınar, E, Radenović, S: Some inevitable remarks on 'Tripled fixed point theorems for mixed monotone Kannan type contractive mappings'. J. Appl. Math. 2014, Article ID 392301 (2014)

11. Al-Mezel, SA, Alsulami, HH, Karapınar, E, Roldán-López-de-Hierro, AF: Discussion on 'Multidimensional coincidence points' via recent publications. Abstr. Appl. Anal. 2014, Article ID 287492 (2014)

12. Erhan, I, Karapınar, E, Roldán-López-de-Hierro, AF, Shahzad, N: Remarks on 'Coupled coincidence point results for a generalized compatible pair with applications.' Fixed Point Theory Appl. 2014, Article ID 207 (2014)

13. Nan, NN, Charoensawan, P: Coupled $g$-coincidence point theorems for a generalized compatible pair in complete metric spaces. Fixed Point Theory Appl. 2014, Article ID 201 (2014)

14. Nan, NN, Charoensawan, $\mathrm{P}:(H, F)$-Closed set and coupled coincidence point theorems for a generalized compatible in partially G-metric spaces. J. Inequal. Appl. 2014, Article ID 342 (2014)

15. Karapınar, E, Roldán, A, Shahzad, N, Sintunavarat, W: Discussion of coupled and tripled coincidence point theorems for $\phi$-contractive mappings without the mixed $g$-monotone property. Fixed Point Theory Appl. 2014, Article ID 92 (2014)

16. Roldán-López-de-Hierro, AF, Shahzad, N: Some fixed/coincidence point theorems under $(\psi, \varphi)$-contractivity conditions without an underlying metric structure. Fixed Point Theory Appl. 2014, Article ID 218 (2014)

17. Kutbi, MA, Roldán, A, Sintunavarat, W, Martínez-Moreno, J, Roldán, C: F-Closed sets and coupled fixed point theorems without the mixed monotone property. Fixed Point Theory Appl. 2013, Article ID 330 (2013)

18. Roldán-López-de-Hierro, AF, Karapınar, E, de la Sen, M: Coincidence point theorems in quasi-metric spaces without assuming the mixed monotone property and consequences in G-metric spaces. Fixed Point Theory Appl. 2014 Article ID 184 (2014)

19. Agarwal, R, Karapınar, E, Roldán, A: Fixed point theorems in quasi-metric spaces and applications to coupled/tripled fixed points on $G^{*}$-metric spaces. J. Nonlinear Convex Anal. (to appear) 